\title{
Effects of structural modification of anti-inflammatory steroidal antedrug on pro-inflammatory mediators and inhibitory cytokines in human alveolar epithelial cells
}

\author{
Gui-Fang Wang ${ }^{1}$, Soonjo Kwon ${ }^{2 *}$, Rakesh Sharma ${ }^{1}$, Hemang Patel ${ }^{2}$, Henry J. Lee ${ }^{3}$ \\ ${ }^{1}$ Chemical and Biomedical Engineering, Florida State University, Tallahassee, USA; soonjo.kwon@usu.edu \\ ${ }^{2}$ Biological Engineering, Utah State University, Logan, USA \\ ${ }^{3}$ Center for Anti-Inflammatory Research, College of Pharmacy and Pharmaceutical Sciences, Florida A\&M University, Tallahassee, \\ USA
}

Received 23 September 2009; revised 19 October 2009; accepted 20 October 2009.

\begin{abstract}
The anti-inflammatory effects of the new steroidal antedrug, 21-acetyloxy-9 $\alpha$-fluoro-11 $\beta$-hydroxyl-3, 20-dioxo-1, 4-pregnadieno-[16 $\alpha, 17 \alpha-d]$ isoxazoline (FP-ISO-21AC), on nitric oxide (NO) and interleukin 8 (IL-8) production, were investigated together with its parent steroid prednisolone (PRED). PRED is one of the anti-inflammatory steroids but has systemic side effects which limit the use of it. PRED was modified with 'antedrug concept' to create safer drugs that attack problems such as inflammation, then quickly become inactive before they can cause systemic side effect. We had a test about the effect of the modified anti-inflammatory steroidal antedrug on anti-inflammatory activity. The present study evaluated their ability to inhibit cytokine-induced NO and IL-8 production in human alveolar epithelial cells. We also investigated their ability to enhance the expression of inhibitory cytokine receptor, interleukin 22 receptor (IL-22R) in human alveolar epithelial cells. Our results showed that FP-ISO-21AC showed higher ability to inhibit the cytokine - induced production of NO than PRED. Exogenous IL-22 was added to the media of both human alveolar epithelial cells (A549) and human lung fibroblast (HLF-1). In the presence of the exogenous inhibitory cytokine IL-22, further reduction of NO production was observed in A549 cells, which express IL-22R, but not in HLF1, which does not express IL-22R. These data suggested that the steroidal antedrugs enhanced the expression of IL-22R. FP-ISO- 21AC showed higher potency than PRED to restore the expression of IL-22R. FP-ISO-21AC further reduced NO production to $27 \%$ and PRED
\end{abstract}

further reduced NO production to $39 \%$. In conclusion, a synthesized steroidal antedrug FP. ISO-21AC showed higher anti-inflammatory effects than PRED by inhibiting the expression of pro-inflammatory mediator NO and stimulating the expression of IL-22R.

Keywords: Steroidal Antedrug; NO Production; IL-8 Production; Anti-Inflammatory Cytokine Receptor; IL-22 Receptor

\section{INTRODUCTION}

Glucocorticoid is one of the most commonly and effectively used drugs to relieve inflammation [1-4]. However, these drugs display a number of serious systemic side effects such as suppression on pituitary-adrenal axis and on the immune system, aggravation of diabetes, hypertension, retardation of growth in children and osteoporosis. These systemic side effects limit the clinical use of corticosteroids [5-9]. Therefore, the main focus of present research is to modify the structure of corticosteroids by reducing the systemic side effects without losing their anti-inflammatory function. A novel strategy was employed by incorporating of metabolically labile functional group onto the steroid chain structure based on the 'antedrug concept'. As Lee et al. described in previous papers, antedrug is defined as a compound which acts locally on the target tissue and it is rapidly metabolized to an inactive metabolite through enzymatic reaction upon entry into the systemic circulation [10-13].

The new anti-inflammatory steroidal antedrug, 21-Acetyloxy-9 $\alpha$-fluoro-11 $\beta$-hydroxy-3, 20-dioxo-1, 4-pregnadieno-[16 $\alpha, 17 \alpha-\mathrm{d}]$ isoxazoline (FP-ISO-21AC) with $\mathrm{C}-16,17$-isoxazoline ring systems was synthesized and its pharmaceutical activities were evaluated [14]. Their study also showed that FP-ISO-21 AC has high binding 
affinities to the glucocorticoid receptor prepared from liver cytosol and exhibited its enhanced inhibitory effects on LPS- induced nitric oxide (NO) production in murine macrophage cells (RAW 264.7)[14]. Although this new steroidal antedrug showed more potential anti-inflammatory effects, few main mechanisms by which multiple pro-inflammatory pathways are switched off and antiinflammatory pathways are switched on in asthma following pretreatment of steroidal antedrugs have been identified. In present time, multiple pro-inflammatory mediators have been implicated in asthma. However, the inhibitory mechanisms of the inflammatory process still remain to be investigated. There is increasing evidence that certain cytokines have anti-inflammatory or immunomodulatory effects and that their secretion might be defective in asthmatic patients. As a result, anti- inflammatory cytokines (inhibitory cytokines) can block the inflammatory process or suppress the intensity of the inflammatory cascade. The severity and the persistence of asthma depend on the "balance" between the proinflammatory cytokines and the anti-inflammatory cytokines [15]. The expression of anti-inflammatory cytokines can be restored by the treatment with steroids or theophylline [16].

In this study, we had a test on the effect of the modified anti-inflammatory steroid (FP-ISO-21AC, isoxazoline derivative, anti-inflammatory steroidal antedrugs) on anti-inflammatory activities. The present study addresses the following fundamental questions: 1) Does structural modification of anti-inflammatory steroidal antedrugs increase the therapeutic index of potent corticosteroids with reducing their systemic side effects? 2) Do these anti-inflammatory steroidal antedrugs enhance or restore the expression of anti-inflammatory proteins (e.g. inhibitory cytokines)? More specifically, the effect of FP-ISO-21AC, an active synthetic derivative of antiinflammatory steroidal antedrug, was evaluated on reduced production of pro-inflammatory mediators (IL-8 and NO). Simultaneously, FP-ISO-21AC was evaluated on enhanced expression of anti-inflammatory cytokine receptor IL-22R in human alveolar epithelial cells. FPISO-21AC showed higher potency to restore the expression of IL-22R and further reduced NO production, compared with PRED. A synthesized steroidal antedrug FPISO-21AC showed higher anti-inflammatory effects than PRED by inhibiting the expression of pro-inflammatory mediator, NO and stimulating the expression of the inhibitory cytokine receptor, IL-22R. The study has enormous diagnostic implications in asthmatic patients and designing novel synthetic anti-inflammatory steroids with less systemic side effects.

\section{MATERIALS AND METHODS}

\subsection{Chemicals and Reagents}

Recombinant human interleukin-1 $\beta$ (IL-1 $\beta$ ), recombi-

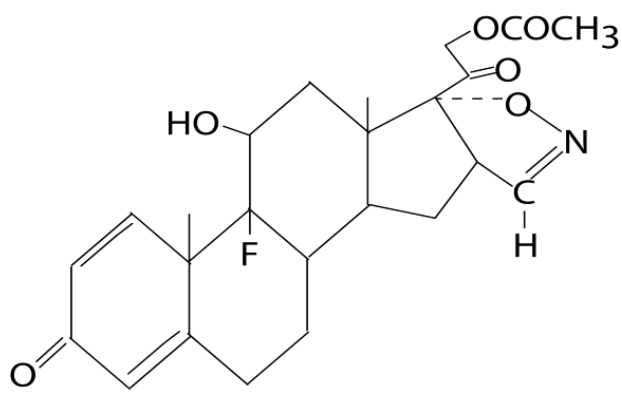

Figure 1. Structure of antedrug FP-ISO-21AC.

nant human tumor necrosis factor-a (TNF- $\alpha$ ), recombinant human interferon- $\gamma$ (IFN- $\gamma$ ) and recombinant human interleukin-22 (IL-22) were obtained from R\&D systems, Inc (Minneapolis, USA). New steroidal antedrug, 21 Acetyloxy-9 $\alpha$-fluoro-11 $\beta$-hydroxy-3, 20-dioxo-1, 4-pregnadieno-[16 $\alpha, 17 \alpha-\mathrm{d}]$ isoxazoline (FP-ISO-21AC) was synthesized as described previously [17-19]. The structure of FP-ISO-21 AC is shown in Figure 1.

\subsection{Cell Culture}

Human alveolar epithelial cells, A549 (ATCC, CCL- 185) and human lung fibroblast, HFL1 (ATCC, CCL- 183) were obtained from ATCC (Manassas, VA, USA). The cells were grown on Ham's F12K medium with $2 \mathrm{mM}$ of L-glutamine adjusted to contain $1.5 \mathrm{~g} / \mathrm{L}$ sodium bicarbonate, $90 \%$ (ATCC, Manassas, VA, USA); and supplied with 10\% fetal bovine serum (ATCC, Manassas, VA, USA). The cells were plated onto $75 \mathrm{~cm}^{2}$ flasks and incubated at $37{ }^{\circ} \mathrm{C}$ with $95 \%$ air and $5 \% \mathrm{CO}_{2}$. Once the cells reached confluence, cells were typsinized with a solution containing $0.25 \%(\mathrm{w} / \mathrm{v})$ trypsin and $0.038 \%$ (w/v) EDTA-4Na (Invitrogen, Carlsbad, California, USA). Cells were then transferred to 24-well plates at the seeding density of 1 X $10^{4}$ per well. When the cells reached confluence (approximately 7 days), they were washed twice with PBS (Phosphate Buffered Saline).

\subsection{Stimulation with Cytokines and/or Steroidal Antedrugs}

Once confluent, cells were incubated in serum-free media for $24 \mathrm{~h}$ before exposure to cytokine(s) and/or steroidal antedrugs. Cells were then pre-incubated for $1 \mathrm{~h}$ with PRED and FP-ISO-AC at $1 \mu \mathrm{M}$ in cell culture medium without serum. After washing cell layers with PBS twice, cells were stimulated with Cytomix (IL-1 $\beta$, TNF$\alpha$ and IFN- $\gamma$ ) at $50 \mathrm{ng} / \mathrm{ml}$ each for $23 \mathrm{~h}$ at $37^{\circ} \mathrm{C}$ in cell culture medium in combination with anti-inflammatory cytokine (IL-22) at $20 \mathrm{ng} / \mathrm{ml}$. Following cytokine exposure, serum was returned to the culture media. NO and IL-8 was analyzed at $24 \mathrm{~h}$ following stimulation.

\subsection{Measurement of Nitric Oxide (NO)}

NO production was measured by NO Analyzer (inNO, 
Nitric Oxide Measuring System, Harvard apparatus, Holliston, Massachusetts, USA). NO sensors were covered with a series of permeable membranes allowing only NO to diffuse through from the sample solution and react at the electrode surface, where it is oxidized. The exchange of electrons resulted in an electrical current which depends on the concentration of NO. The concentration of NO in the sample is proportional to the amount of NO that diffused through the membranes. Consequently, the electrical current is proportional to the concentration of NO in the sample. The NO data were also confirmed by Griess reagent system (Promega Corporation, WI).

\subsection{Measurement of IL-8 Expression}

IL-8 released into culture medium was measured with an enzyme-linked immunosorbent assay (ELISA) sandwich kit (ELISA quantikine, ref. D8050, R\&D Systems, Inc, Minneapolis, USA). Colorimetric changes were measured in a microplate reader (BioRad) at $490 \mathrm{~nm}$. The IL8 level was estimated by interpolation from the calibration curve obtained using the standard IL-8.

\section{RESULTS}

PRED was structurally modified with 'antedrug concept' to reduce the systemic side effects [14]. A synthesized FP-ISO-21AC showed higher ability to inhibit the cytokine-induced production of NO than PRED. There was no significant difference between FP-ISO-21AC and PRED on inhibitory effect on cytokine-induced IL-8 expression, but the FP-ISO-21AC showed higher inhibitory effect on cytokine-induced NO production (Figure 2) and also enhanced IL-22R expression (Figure 4A) with less systemic side effects.

\subsection{Anti-Inflammatory Steroidal Antedrug, FP-ISO-21AC, and Its Parent PRED Inhibit Cytokine-Induced NO Production}

Figure 2 shows the inhibitory effects of anti-inflammatory steroidal antedrugs, FP-ISO-21AC and its parent prednisolone (PRED), on cytokine-induced NO production. Both FP-ISO-21AC and PRED inhibited cytokine-induced NO production. One hour pre-treatment of FP-ISO-21AC at $1 \mu \mathrm{M}$ reduced cytokine-induced NO production down to $37 \%$. PRED at $1 \mathrm{M}$ reduced cyto kine-induced NO production down to $65 \%$. The FP-ISO$21 \mathrm{AC}$ showed higher inhibitory effect on cytokine-induced NO production. In other words, the FP-ISO-21AC showed higher anti-inflammatory effect.

\subsection{Anti-Inflammatory Steroidal Antedrug FP-ISO-21AC and Its Parent PRED Inhibit Cytokine-Induced Interleukin-8 (IL-8) Production}

Anti-inflammatory steroidal antedrug FP-ISO-21AC and

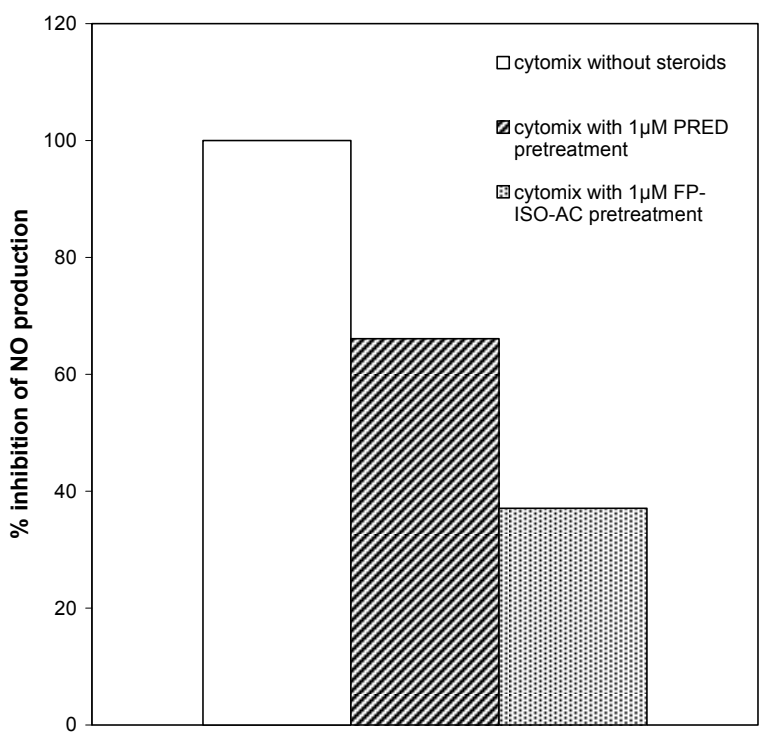

Figure 2. Inhibitory effect of steroids on cytokine-induced NO production in A549 human alveolar epithelial cells. Cells were exposed cytomix (The mixture of the three cytokines IL-1 $\beta$, TNF- $\alpha$ and IFN- $\gamma$ and the concentration of each cytokine is 50 $\mathrm{ng} / \mathrm{ml}$ ) following pretreatment of steroids at indicated concentrations for 1 hour. The concentration of NO was measured 24 hours after cytokine exposure by amiNO series of NO sensors. Data from steroids treatment experiments were expressed as percentage of untreated controls. $\mathrm{p} \leq 0.05$.

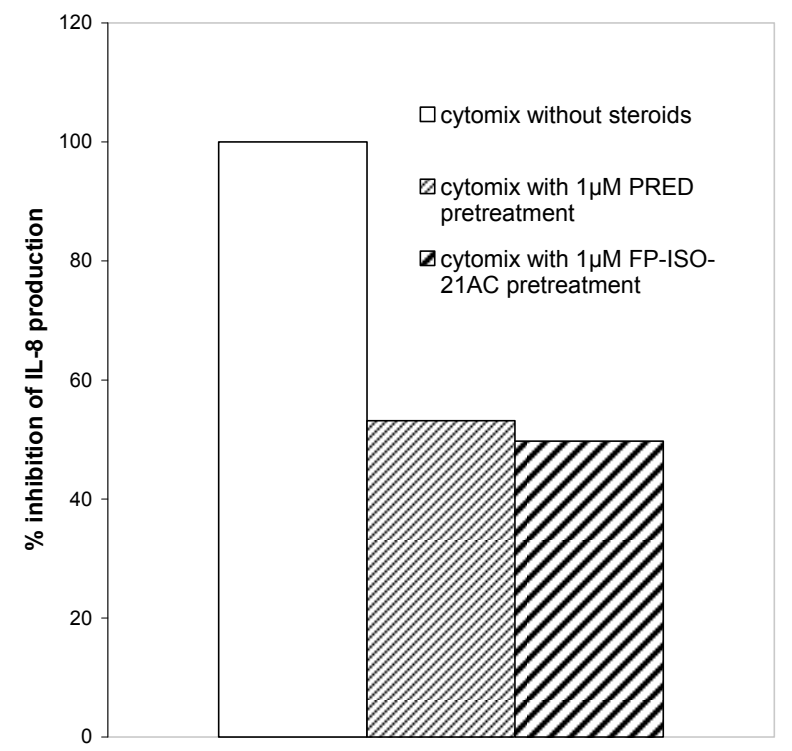

Figure 3. Inhibitory effect of steroids on cytokine-induced IL-8 production in A549 human alveolar epithelial cells. Cells were exposed cytomix (IL-1 $\beta, \mathrm{TNF}-\alpha$ and IFN- $\gamma$ at $50 \mathrm{ng} / \mathrm{ml}$ respectively) following pretreatment of steroids at indicated concentrations for 1 hour. The concentration of IL-8 was measured by ELISA after 24 hours exposure to cytokine. Data from steroids treatment experiments were expressed as percentage of untreated controls. $\mathrm{p} \leq 0.05$. One hour pretreatment of FP-ISO-21AC and PRED at $1 \mu \mathrm{M}$ reduce cytokine-induced IL-8 production to $50 \%$ and $53 \%$ respectively. 
PRED inhibited cytokine-induced IL-8 production (Figure 3). The data showed that one hour pretreatment of FP-ISO-21AC at $1 \mu \mathrm{M}$ reduced cytokine-induced IL-8 production down to $50 \%$. PRED reduced cytokine - induced IL- 8 production down to $53 \%$. There was no significant difference between FP-ISO-21AC and PRED on the inhibitory effect on cytokine-induced IL-8 expression.

\subsection{Effects of Anti-Inflammatory Steroidal Antedrug on Inhibitory Cytokine}

As shown in Figure 4, PRED and FP-ISO-21AC significantly reduced the cytokine-induced NO production. One hour pre-treatment of PRED and FP-ISO-21AC at 1 $\mu \mathrm{M}$ reduced cytokine-induced NO production down to $57 \%$ and $43 \%$, respectively. In the presence of exogenous IL-22, a further reduction about 17\% (39\% and $26 \%$, respectively for FP-ISO-21AC and PRED) of cytokine-induced NO production in A549 cells pre-treated with anti-inflammatory steroids (Figure 4A) was observed. Interestingly, there was no significant effect of exogenous IL-22 on anti-inflammatory steroids in cytokine-induced NO production from HLF1 (Figure 4B).

\section{DISCUSSION}

Asthma is a chronic, episodic disease of the airways characterized by repeated exacerbations of bronchial airway narrowing and difficult breathing. Chronic and persistent bronchial asthma is life-threatening and distresses more than $5 \%$ of the population in the United States alone and the incidence has been increasing since the 1980's [20,21]. In asthma treatment, consistent efforts have been made in searching for effective steroids with least side effects. In this direction, a considerable amount of research has been conducted to increase the therapeutic index of potent corticosteroids and reducing their systemic side effects. Notably, FP-ISO-21AC was designed with antedrug concept and was shown to have less systemic side effects [10]. It has higher anti-inflammatory activities than parent steroid PRED $[14,17$, 18]. Corticosteroids are recently shown to inhibit the expression of pro-inflammatory mediators such as $\mathrm{NO}$ [22], IL-8 [23,24] and IL-5 [24], with their stimulated release of the anti-inflammatory cytokine such as IL-10 [25].

However, it is unclear whether structural modification of corticosteroids may affect these inhibitory pathways at sites of inflammation. The present study is an attempt to better understand the antedrug switch off multiple pro-inflammatory pathways and switch on anti-inflammatory pathways in asthma with further investigations on NO production. In this study, anti-inflammatory steroidal antedrug FP-ISO-21AC, which was derived from PRED was used to test its effects on inhibiting NO pro-
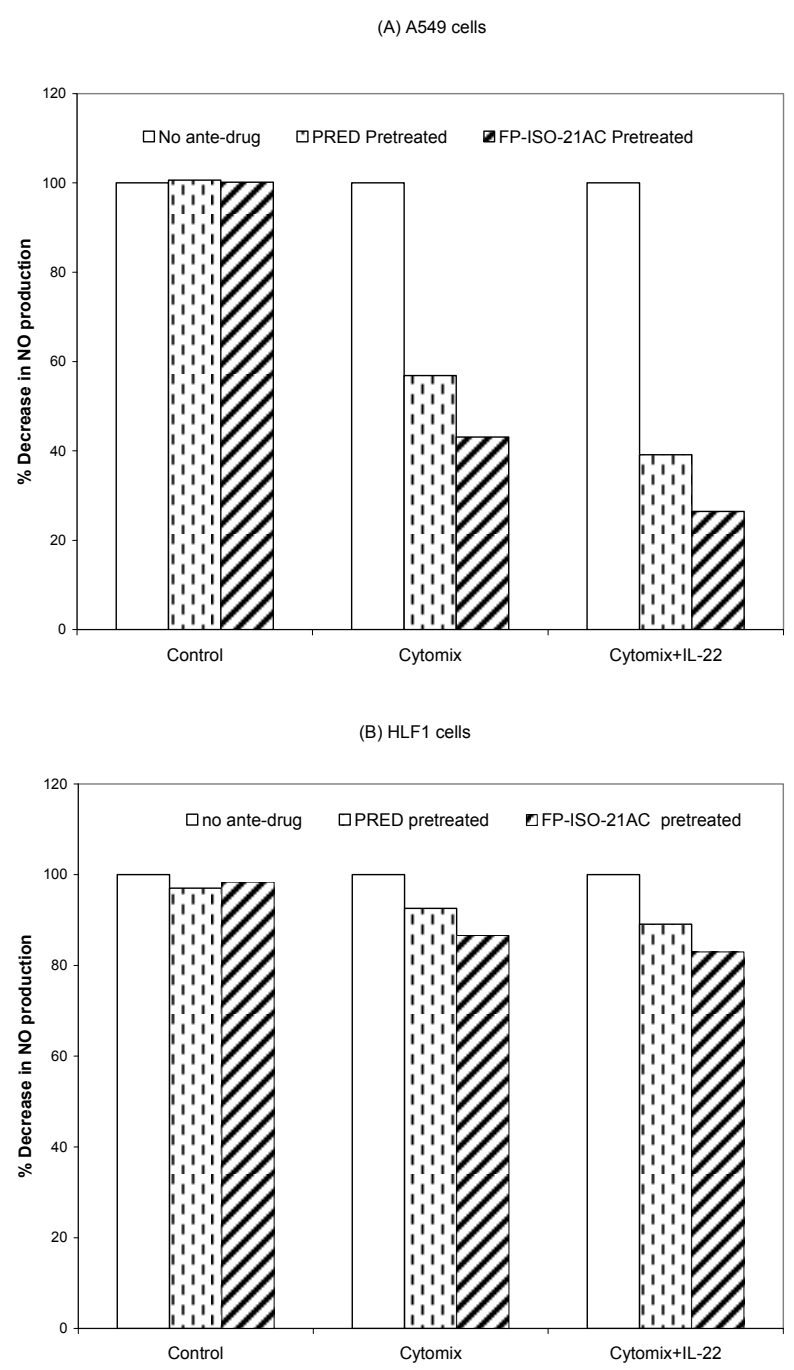

Figure 4. (A) Effects of anti-inflammatory steroidal antedrug FP-ISO-21AC and PRED on inhibitory cytokine in cytokine-induced NO production in A549 cells. FP-ISO-21AC and PRED further reduced cytokine induced NO production in the presence of exogenous IL-22. (B) Effects of anti-inflammatory steroidal antedrug FP-ISO-21AC and PRED on inhibitory cytokines in cytokine-induced NO production in HLF1. No further reduced cytokine induced NO production was observed in the presence of exogenous IL-22. Cells were stimulated with three cytokines (IL- $1 \beta, \mathrm{TNF}-\alpha$, and IFN- $\gamma, 50$ $\mathrm{ng} / \mathrm{mL}$ respectively) or three cytokines (IL- $1 \beta, \mathrm{TNF}-\alpha$, and IFN- $\gamma, 50 \mathrm{ng} / \mathrm{mL}$ respectively) and IL-22 $(20 \mathrm{ng} / \mathrm{mL})$ in combination. After 24 hours incubation, nitric oxide was measured. Data from steroids treatment experiment were expressed as percentage of untreated controls, $\mathrm{p} \leq 0.05$.

duction in human alveolar epithelial cells (A549). Our results show that both FP-ISO-21AC and PRED inhibited the cytokine-induced NO production. One hour pretreatment of FP-ISO-21-AC at $1 \mu \mathrm{M}$ reduced cytokineinduced NO production down to $37 \%$. The PRED showed lower inhibitory effect than FP-ISO-21AC and 
reduced cytokine-induced NO production down to $65 \%$ (Figure 2). There was no significant difference between FP-ISO-21AC and PRED on inhibitory effect on cytokine-induced IL-8 expression, but the FP-ISO-21AC showed higher inhibitory effect on cytokine-induced NO production (Figure 2).

To confirm the observed decrease in NO production related to enhanced expression of inhibitory cytokines, exogenous IL-22 was added to the media of A549 in the presence or absence of anti-inflammatory steroids. IL-22 is a member of the human type I IFN family, which includes IL-10. Our data corroborates with earlier report on IL-22 effect in the presence of inflammatory steroids [26]. Other studies support our observations on lung epithelial cells (A549) which express IL-22R1 (interleukin 22 receptor) subunit in response to IL-22 [15]. As the results indicated in Figure $4 \mathrm{~A}$ and $4 \mathrm{~B}$, both PRED and FP-ISO-21AC may enhance the expression of IL-22R1, and increase the responsiveness to exogenous IL-22 in A549 cells which do express IL-22R1.

An important factor is the balance between the effects of pro-inflammatory and anti-inflammatory chemokines or cytokines in determining the severity of the inflammatory disease. Corticosteroids have effects on expression of cytokines receptors [27,28]. Corticosteroids are understood to upregulate cytokine receptor expression that correlates with enhanced cytokine effects on target cells [27]. Other earlier studies also suggest that receptors for IL-1 [29,30], IL-2 [31], IL-4 [32], IL-6 [33,34], IFN- $\gamma$ [35] and GM-CSF [36] are all up-regulated by glucocorticoids.

Inflammatory stimuli increase the expression of many inflammatory genes, resulting in the inflammatory responses. The same stimuli may also induce inhibitory cytokines, which can inhibit the expression of these inflammatory proteins, thus terminating this inflammatory response. There has been increasing evidence that a defective expression of inhibitory cytokines is observed in patients with asthma [37]. The reduced expression of inhibitory cytokines leads to increased and more prolonged inflammation. Subsequent treatment by anti-inflammatory steroids can restore the secretion of inhibitory cytokines [37]. Structural modification of corticosteroids may restore this defective expression of inhibitory cytokines. The structural modification can be an important direction forwards the design of novel anti-inflammatory steroidal antedrugs for the treatment of asthma.

There has been increasing evidences that the role of inhibitory cytokines is crucial on the treatment of asthma [38]. IL-22 is one of the most important inhibitory cytokines involved in the inhibition of the inflammation. It can regulate the expression of proinflammatory cytokines [39]. However, the effect of steroid on IL-22R is not known. In order to test if steroids can enhance the expression of IL-22R, the effects of IL-22 on the inhibition of NO production induced by the pre-treatment of anti-inflammatory steroids was tested. Our data demonstrated that in the presence of the exogenous inhibitory cytokine IL-22, further reduction of NO production was observed in A549 cells (Figure 4A) as earlier demonstrated to express IL-22R [39] but not in HLF1 (Figure 4B) which do not express IL-22R. These data suggested that the steroidal ante-drugs may enhance the expression the IL-22 receptor. FP-ISO-21AC showed higher potency than PRED to restore the expression of IL-22R. FP-ISO-21AC further reduced NO production to $27 \%$ and PRED further reduced NO production to $39 \%$.

In conclusion, a synthesized antedrug FP-ISO-21AC showed higher anti-inflammatory effects than PRED by inhibiting the expression of pro-inflammatory mediator NO. There was no significant difference between FP-ISO-21AC and PRED on inhibitory effect on cytokine-induced IL-8 expression, but the FP-ISO-21AC showed higher inhibitory effect on cytokine-induced NO production. FP-ISO-21AC also showed higher potency than its parent PRED in stimulating the possible expression of IL-22R. This study showed that structural modification of anti-inflammatory steroids with 'antedrug concept' (with the direction of reducing the systemic side effects) could enhance their anti-inflammatory activities with less systemic side effects.

\section{REFERENCES}

[1] D. T. Boumpas, G. P. Chrousos, R. L. Wilder, T. R. Cupps, J. E. Balow, (1993) Glucocorticoid therapy for immunemediated diseases - basic and clinical correlates, Annals of Internal Medicine, 119, 1198-1208.

[2] J. W. Funder, (1997) Glucocorticoid and mineralocorticoid receptors: biology and clinical relevance, Annual Review of Medicine, 48, 231-240.

[3] H. M. Reichardt, G. Schutz, (1998) Glucocorticoid signalling - multiple variations of a common theme, Molecular and Cellular Endocrinology, 146, 1-6.

[4] L.I. McKay, JA. Cidlowski, (1999) Molecular control of immune/inflammatory responses: Interactions between nuclear factor-kappa B and steroid receptor-signaling pathways, Endocrine Reviews, 20, 435-459.

[5] L. A. Wolfraim, (2006) Treating autoimmune diseases through restoration of antigen-specific immune tolerance, Archivum Immunologiae Et Therapiae Experimentalis, 54, 1-13.

[6] H. Schacke, W. D. Docke, K. Asadullah, (2002) Mechanisms involved in the side effects of glucocorticoids, Pharmacology \& Therapeutics, 96, 23-43.

[7] E. Garbe, J. LeLorier, J. F. Boivin, S. Suissa, (1997) Risk of ocular hypertension or open-angle glaucoma in elderly patients on oral glucocorticoids, Lancet, 350, 979-982.

[8] P. B. Jacobson, T. W. von Geldern, L. Ohman, M. Osterland, J. H. Wang, B. Zinker, D. Wilcox, PT. Nguyen, A. Mika, S. Fung, T. Fey, A. Goos-Nilsson, M. Grynfarb, T. 
Barkhem, K. Marsh, D. W. A. Beno, B. Nga-Nguyen, P. R. Kym, J. T. Link, N. Tu, DS. Edgerton, A. Cherrington, S. Efendic, B. C. Lane, T. J. Opgenorth, (2005) Hepatic glucocorticoid receptor antagonism is sufficient to reduce elevated hepatic glucose output and improve glucose control in animal models of type 2 diabetes, Journal of Pharmacology and Experimental Therapeutics, 314, 191200.

[9] J. Nishimura, S. Ikuyama, (2000) Glucocorticoid-Induced osteoporosis: pathogenesis and management, Journal of Bone and Mineral Metabolism, 18, 350-352.

[10] H. J. Lee, MRI. Soliman, (1982) Anti-Inflammatory steroids without pituitary-adrenal suppression, Science, 215 , 989-991.

[11] H. J. Lee, M. A. Khalil, J. W. Lee, (1984) Antedrug - a conceptual basis for safer anti-inflammatory steroids, Drugs under Experimental and Clinical Research, 10, 835-844.

[12] H. J. Lee, R. Trottier, (1980) Antiinflammatory activity of two novel derivatives of prednisolone, Res Commun Chem Pathol Pharmacol, 27, 611-4.

[13] H. Ueno, A. Maruyama, M. Miyake, E. Nakao, K. Nakao, K. Umezu, I. Nitta, (1991) Synthesis and evaluation of antiinflammatory activities of a series of corticosteroid 17-Alpha-Esters containing a Functional-Group, Journal of Medicinal Chemistry, 34, 2468-2473.

[14] K. K. Park, D. H. Ko, Z. You, M. O. F. Khan, H. J. Lee, (2006) In vitro anti-inflammatory activities of new steroidal antedrugs: [16 alpha, 17 alpha-d] isoxazoline and [16 alpha, 17 alpha-d]-3'-hydroxy-iminoformyl isoxazoline derivatives of prednisolone and 9 alpha - fluoroprednisolone, Steroids, 71, 183-188.

[15] H. A. Whittington, L. Armstrong, K. M. Uppington, A.B. Millar, (2004Interleukin-22 - A potential immunomodulatory molecule in the lung, American Journal of Respiratory Cell and Molecular Biology, 31, 220-226.

[16] B. M. Necela, J. A.Cidlowski, (2003) Crystallization of the human glucocorticoid receptor ligand binding domain: a step towards selective glucocorticoids, Trends in Pharmacological Sciences, 24, 58-61.

[17] M. A. Khalil, M. F. Maponya, D. H. Ko, Z. Q. You, E. T. Oriaku, H. J. Lee, (1996) New anti-inflammatory steroids: [16 alpha, 17 alpha-d] isoxazoline derivatives of prednisolone and 9 alpha-fluoroprednisolone, Medicinal Chemistry Research, 6, 52-60.

[18] D. H. Ko, M. F. Maponya, M. A. Khalil, E. T. Oriaku, Z. Q. You, H. J. Lee, (1997) New anti-inflammatory steroids: [16 alpha, 17 alpha-d] -3'-hydroxyiminoformyl isoxazoline derivatives of prednisolone and 9 apha- flu roprednisolone. Medicinal Chemistry Research, 7, 313-323.

[19] T. Kwon, A. S. Heiman, E. T. Oriaku, K. Yoon, H. J. Lee (1995) New steroidal antiinflammatory antedrugs - steroidal [16- Alpha,17-Alpha-D]-3'-Carbethoxyisoxazolines. Journal of Medicinal Chemistry, 38, 1048-1051.

[20] JA. Elias, Z. Zhu, G. Chupp, RJ. Homer, (1999) Airway remodeling in asthma, Journal of Clinical Investigation; 104, 1001-1006.

[21] J. Elias, (1999) Inspirations on asthma, Journal of Clinical Investigation, 104, 827-827.

[22] N. K. Worrall, T. P. Misko, P. M. Sullivan, J. J. Hui, C. P. Rodi, T. B. Ferguson, (1996) Corticosteroids inhibit expression of inducible nitric oxide synthase during acute cardiac allograft rejection, Transplantation, 61, 324-328.

[23] K. Nyhlen, M. Linden, R. Andersson, S. Uppugunduri, (2000) Corticosteroids and interferons inhibit cytokine-induced production of IL-8 by human endothelial cells, Cytokine, 12, 355-360.

[24] B. Wallwork, W. Coman, F. Feron, A. Mackay-Sim, A. Cervin, (2002) Clarithromycin and prednisolone inhibit cytokine production in chronic rhinosinusitis, Laryngoscope, 112, 1827-1830.

[25] M. John, S. Lim, J. Seybold, P. Jose, A. Robichaud, B. O'Connor, P. J. Barnes, K. F. Chung, (1998) Inhaled corticosteroids increase interleukin-10 but reduce macrophage inflammatory protein-1 alpha, granulocyte-macrophage colony-stimulating factor, and interferon-gamma release from alveolar macrophages in asthma, American Journal of Respiratory and Critical Care Medicine, 157, 256-262.

[26] L. Dumoutier, J. Louahed, J. C. Renauld, Cloning and characterization of IL-10-related T cell-derived inducible factor (IL-TIF): a novel cytokine structurally related to IL-10 and inducible by IL-9, Journal of Immunology 12000, 164, 1814-1819.

[27] W. Y. Almawi, H. N. Beyhum, A. A. Rahme, M. J. Rieder, (1996) Regulation of cytokine and cytokine receptor expression by glucocorticoids, Journal of Leukocyte Biology, 60, 563-572.

[28] G. J. Wiegers, J. M. H. M. Reul, (1998) Induction of cytokine receptors by glucocorticoids: functional and pathological significance, Trends in Pharmacological Sciences, 19, 317-321.

[29] T. Akahoshi, J. J. Oppenheim, K. Matsushima, (1988) Induction of high-affinity Interleukin-1 receptor on human peripheral-blood lymphocytes by glucocorticoid hormones, Journal of Experimental Medicine, 167, 924936.

[30] P. E. Gottschall, K. Koves, K. Mizuno, I. Tatsuno, A. Arimura, (1991) Glucocorticoid up-regulation of Interleukin-1 receptor expression in a glioblastoma Cell-Line,. American Journal of Physiology, 261, E362-E368.

[31] G. J. Wiegers, M. S. Labeur, I. E. M. Stec, W. E. F. Klinkert, F. Holsboer, J. M. H. M. Reul, (1995) Glucocorticoids accelerate anti-T cell receptor-induced T-cell growth, Journal of Immunology, 155, 1893-1902.

[32] R. L. K. Paterson, R. Or, J. M. Domenico, G. Delespesse, E. W. Gelfand, (1994) Regulation of Cd23 expression by Il-4 and corticosteroid in human B-lymphocytes - altered response after Ebv infection, Journal of Immunology, 152, 2139-2147.

[33] L. Snyers, L. Dewit, J. Content, (1990) Glucocorticoid up-regulation of high-affinity Interleukin-6 receptors on human Epithelial-Cells, Proceedings of the National Academy of Sciences of the United States of America, 87, 2838-2842.

[34] S. P. Campos, Y. P. Wang, A. Koj, H. Baumann, (1993) Divergent transforming Growth-Factor-Beta effects on Il-6 regulation of Acute-Phase Plasma-Proteins in Rat Hepatoma-Cells, Journal of Immunology, 151, 7128-7137.

[35] R. W. Strickland, L. M. Wahl, D. S. Finbloom, (1986) Corticosteroids enhance the binding of recombinant interferon-gamma to cultured human-monocytes, Journal of Immunology, 137, 1577-1580.

[36] C. M. Hawrylowicz, L. Guida, E. Paleolog, (1994) Dexamethasone up-regulates granulocyte-macrophage colony 
- stimulating ractor-receptor expression on human monocytes, Immunology, 83, 274-280.

[37] P. J. Barnes, S. Lim, (1998) Inhibitory cytokines in asthma, Molecular Medicine Today, 4, 452-458.

[38] D. Kunz, G. Walker, W. Eberhardt, J. Pfeilschifter, (1996) Molecular mechanisms of dexamethasone inhibition of nitric oxide synthase expression in interleukin 1 betastimulated mesangial cells: evidence for the involvement of transcriptional and posttranscriptional regulation,
Proceedings of the National Academy of Sciences of the United States of America, 93, 255-259.

[39] D. A. Geller, A. K. Nussler, M. Disilvio, C. J. Lowenstein, R. A. Shapiro, S. C. Wang, R. L. Simmons, T. R. Billiar, (1993) Cytokines, endotoxin, and glucocorticoids regulate the expression of inducible Nitric-Oxide synthase in hepatocytes, Proceedings of the National Academy of Sciences of the United States of America, 90, 522-526. 